\title{
NUMERICAL SIMULATION OF BIOCHEMICAL BEHAVIOUR OF BIOSENSORS WITH PERFORATED MEMBRANE
}

\author{
Romas Baronas \\ Institute of Mathematics and Informatics \\ Vilnius University \\ Naugarduko 24, Vilnius 03225, Lithuania \\ E-mail: romas.baronas@mif.vu.lt
}

\section{KEYWORDS}

Modelling, reaction-diffusion, simulation, biosensors.

\begin{abstract}
A two-dimensional-in-space mathematical model of amperometric biosensors with selective and perforated membranes has been proposed and analyzed. The model involves the geometry of holes partially or fully filled with an enzyme. The model is based on a system of the reaction-diffusion equations containing a nonlinear term related to the Michaelis-Menten enzymatic reaction. Using numerical simulation of the biosensor action, the influence of the geometry of the holes as well as of the filling level of the enzyme in the holes on the biosensor response was investigated. The numerical simulation was carried out using the finite difference technique. The calculation demonstrated that the effect of the filling level on the biosensor response decreases with decrease of radius of the holes.
\end{abstract}

\section{INTRODUCTION}

Biosensors are sensing devices made up of a combination of a specific biological element, usually the enzyme, that recognizes a specific analyte and the transducer that translates the biorecognition event into an electrical signal (Turner et al. 1987; Scheller and Schubert 1992). The signal is proportional to the concentration of the target analyte-substrate. The amperometric biosensors measure the faradic current that arises on a working indicator electrode by direct electrochemical oxidation or reduction of the product of the biochemical reaction. The amperometric biosensors are known to be reliable, cheap and highly sensitive for environment, clinical and industrial purposes (Rogers 1995; Wollenberger et al. 1997).

A practical biosensor contains a multilayer enzyme membrane (Scheller and Schubert 1992; Baeumner 2004). The electrode acting as a transducer of the biosensor is covered by a selective membrane, following a layer of immobilized enzyme and an outer membrane. To improve the efficiency of biosensors design and to optimize the biosensors configuration a model of the real biosensor should be build (Ferreira et al. 2003). The modelling of biosensors with perforated membrane has been performed by Schulmeister and Pfeiffer (1993). Very recently, an improved model taking into consideration the geometry of the membrane perforation has been proposed (Baronas et al. 2006). In this model, the holes in the perforated membrane were assumed fully filled with an enzyme.

The task of this investigation was to build a model approaching the real amperometric biosensor where the holes in perforated membrane are partially filled with the enzyme. By changing input parameters the output results were numerically analyzed with a special emphasis to the influence of the geometry of the perforated membrane and of the external diffusion region to the biosensor response. The numerical simulation was carried out using the finite difference technique (Britz 1988; Samarskii 2001).

\section{PRINCIPAL STRUCTURE}

We assume that the thickness of the perforated membrane as well as of the selective membrane of a biosensor is much less than its length and width. The selective membrane is assumed of a uniform thickness. The holes in the perforated membrane were modelled by right cylinders of uniform diameter and spacing, forming a hexagonal pattern. Figure 1 presents the biosensor schematically.

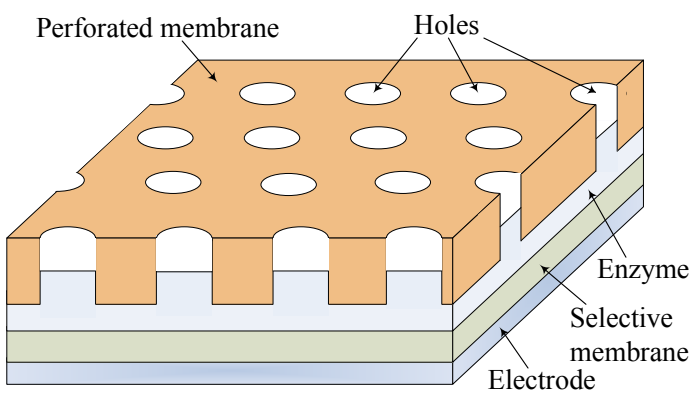

Figure 1: A Principal Structure of a Biosensor.

Due to the uniform distribution of the holes, the entire biosensor may be divided into equal hexagonal prisms with regular hexagonal bases (Baronas et al. 2006). For simplicity, it is reasonable to consider a circle whose area equals to that of the hexagon and to regard one of the cylinders as a unit cell of the biosensor. Due to the symmetry of the unit cell, we may consider only a half of the transverse section of the unit cell. 
Figure 2 shows the profile of the unit of the biosensor, represented schematically in Figure 1 . In Figure 2, $\Omega_{1}$ represents the selective membrane, $\Omega_{2}$ corresponds to the enzyme region, $\Omega_{3}$ stands for to the external diffusion layer, $a_{2}$ is the radius of the base of the unit cell, $a_{1}$ is the radius of the holes, $b_{1}$ stands for the thickness of the selective membrane, $b_{4}-b_{2}$ is the thickness the perforated membrane, $b_{5}-b_{4}$ is the thickness of the diffusion layer. We assume that the holes are partially filled with the enzyme, $b_{3}$ stands for the filling level.

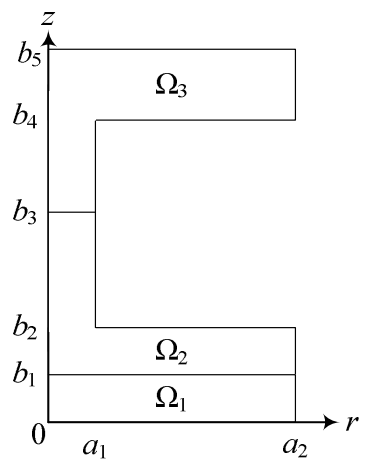

Figure 2: The Profile of the Unit Cell.

\section{MATHEMATICAL MODEL}

The mathematical model of a biosensor with the selective and perforated membranes (Figures 1 and 2) may be formulated in a two-dimensional domain consisting mainly of three regions: the selective membrane $\Omega_{1}$, the enzyme region $\Omega_{2}$ and the region $\Omega_{3}$ of the external diffusion. In selective membrane only the mass transport by diffusion of the reaction product takes place. In the enzyme region we consider the mass transport by diffusion and the enzyme-catalysed reaction

$$
\mathrm{S}+\mathrm{E} \leftrightarrow \mathrm{ES} \rightarrow \mathrm{E}+\mathrm{P}
$$

In this scheme the substrate (S) combines reversibly with an enzyme (E) to form a complex (ES) (Scheller and Schubert 1992). The complex then dissociates into a product $(\mathrm{P})$ and the enzyme is regenerated. In the region $\Omega_{3}$ the mass transport by diffusion of the substrate as well as of the product takes place.

Assuming the quasi steady state approximation, the concentration of the intermediate complex (ES) do not change and may be neglected when simulating the biochemical behaviour of biosensors (Turner et al. 1987; Scheller and Schubert 1992). The dynamics of the biosensor, presented schematically in Figures 1 and 2, can be described by the reaction-diffusion system $(t>0)$

$$
\begin{aligned}
& \frac{\partial P_{1}}{\partial t}=D_{1} \Delta P_{1}, \quad(r, z) \in \Omega_{1}, \\
& \frac{\partial S_{2}}{\partial t}=D_{2} \Delta S_{2}-\frac{V_{\max } S_{2}}{K_{M}+S_{2}}, \\
& \frac{\partial P_{2}}{\partial t}=D_{2} \Delta P_{2}+\frac{V_{\max } S_{2}}{K_{M}+S_{2}}, \quad(r, z) \in \Omega_{2},
\end{aligned}
$$

$$
\frac{\partial S_{3}}{\partial t}=D_{3} \Delta S_{3}, \quad \frac{\partial P_{3}}{\partial t}=D_{3} \Delta P_{3}, \quad(r, z) \in \Omega_{3}
$$

where $\Delta$ is the Laplace operator, $P_{i}=P_{i}(r, z, t)$ is concentration of the reaction product, $S_{j}=S_{j}(r, z, t)$ is the substrate concentration, $V_{\max }$ - the maximal enzymatic rate, $K_{M}$ - the Michaelis constant, $i=1,2,3, j$ $=2,3$ (Baronas et al. 2006).

Let $\bar{\Omega}_{i}$ be a closed region, corresponding to the open region $\Omega_{i}$, and $\Gamma$ - the upper boundary of $\bar{\Omega}_{3}, i=1,2$, 3. The initial $(t=0)$ conditions are as follows:

$$
\begin{array}{ll}
S_{2}(r, z, 0)=0, & (r, z) \in \bar{\Omega}_{2}, \\
S_{3}(r, z, 0)=0, & (r, z) \in \bar{\Omega}_{3} \backslash \Gamma, \\
S_{3}(r, z, 0)=S_{0}, & (r, z) \in \Gamma, \\
P_{i}(r, z, 0)=0, & (r, z) \in \bar{\Omega}_{i}, \quad i=1,2,3,
\end{array}
$$

where $S_{0}$ is the concentration of the substrate present in the bulk solution.

The product is an electro-active substance. The electrode potential is chosen to keep zero concentration of the product at the electrode surface $(t>0)$,

$$
P_{1}(r, 0, t)=0, \quad r \in\left[0, a_{2}\right] .
$$

On the boundary $\Gamma$ the concentrations of both species are maintained constant $((t>0)$,

$$
S_{3}\left(r, b_{5}, t\right)=S_{0}, \quad P_{3}\left(r, b_{5}, t\right)=0, \quad r \in\left[0, a_{2}\right] .
$$

Non-leakage conditions were employed for the boundaries of the symmetry of the unit cell and of the perforated membrane. On the boundary between two adjusting regions having different diffusivities we defined the matching conditions requiring the concentration continuity for both species.

The governing equations (1)-(3) together with the initial, boundary and matching conditions form together a boundary value problem.

The anodic current is accepted as a response of amperometric biosensors in physical experiments. The current depends upon the flux of the reaction product at the electrode surface, i.e. at the border $z=0$. Consequently, the density $i(t)\left(\mathrm{A} / \mathrm{m}^{2}\right)$ of the current at time $t$ can be obtained explicitly from Faraday's and Fick's laws using the flux of the product concentration $P_{1}$ at the surface of the electrode,

$$
i(t)=\left.n_{\mathrm{e}} F D_{1} \frac{2}{a_{2}^{2}} \int_{0}^{a_{2}} \frac{\partial P_{1}}{\partial z}\right|_{z=0} r \mathrm{~d} r,
$$

where $n_{\mathrm{e}}$ is a number of electrons involved in a charge transfer at the electrode surface and $F$ is Faraday constant. We assume, that the considering system approaches a steady-state as $t \rightarrow \infty$,

$$
I=\lim _{t \rightarrow \infty} i(t),
$$

where $I$ is the steady-state current. 


\section{NUMERICAL SIMULATION}

Definite problems arise when solving analytically nonlinear partial differential equations in a domain of the complex geometry. Because of this the problem was solved numerically using the finite difference technique (Britz 1988; Samarskii 2001). To find a numerical solution of the problem we introduced a quasi-uniform discrete grid. Using alternating direction method a semiimplicit linear finite difference scheme has been built as a result of the difference approximation. The resulting system of linear algebraic equations was solved rather efficiently because of the tridiagonality of the matrix of the system.

In the digital simulation, the main problem was an overload of calculation due to permissible conditions: $a_{1}<<a_{2}$ and $b_{2}<<b_{4}$ (see Figure 2). To have an accurate and stable result it was required to use very small step size in $z$ direction at the boundaries $z=0$ and $z=b_{5}$. Because of the concavity of angles at points $\left(a_{1}\right.$, $\left.b_{2}\right)$ and $\left(a_{1}, b_{4}\right)$ it was necessary to use very small step size in both space directions: $r$ and $z$ at the boundaries $r$ $=a_{1}, z=b_{2}$ and $z=b_{4}$. Due to the matching conditions between adjacent regions with different diffusivities, we used also small step size at the boundaries $z=b_{1}$ and $z=$ $b_{3}$. We assumed, that farther from all these peculiar boundaries, step size may increase in both space directions: $r$ and $z$. Consequently, in the direction $r$, an exponentially increasing step size was used to both sides from $a_{1}$ : to $a_{2}$ and down to 0 . In the direction $z$, an exponentially increasing step size was used form 0 to $b_{1} / 2$, from $b_{5}$ down to $\left(b_{4}+b_{5}\right) / 2$, from $b_{j}$ down to $\left(b_{j}+\right.$ $\left.b_{j-1}\right) / 2$ and from $b_{j}$ to $\left(b_{j}+b_{j+1}\right) / 2, j=1,2,3,4$, where $b_{0}=$ 0 .

Usually, alternating direction method does not restrict time increment. However, the step size in the direction of time is restricted due to the nonlinear reaction term in equations (2), boundary conditions and the geometry of the domain. In order to achieve accurate and stable solution of the problem, at the beginning of the reactiondiffusion process we employed the restriction condition, which is usually used for fully explicit schemes. Since the biosensor action obeys the steady-state assumption when $t \rightarrow \infty$, it was reasonable to apply an increasing step size in the time direction. The final step size was in a few orders of magnitude higher than the fist one.

The digital simulator has been programmed in JAVA language (Moreira et al. 2000).

\section{RESULTS AND DISCUSSION}

In practice, the upper boundary of the enzyme in the holes varies between the bottom and the top of the perforated membrane, i.e. $b_{3}$ varies from $b_{2}$ to $b_{4}$.

The steady state biosensor current is very sensitive to changes of the maximal enzymatic rate $V_{\max }$ and substrate concentration $S_{0}$ (Baronas et al. 2003; Baronas et al. 2004; Scheller and Schubert 1992). Changing values of these two parameters the steady state current varies even in orders of magnitude. Because of this, we calculate the biosensor response at different values of $V_{\max }$ and $S_{0}$. To evaluate the effect of holes filling with the enzyme on the biosensor response we normalize the steady-state current,

$$
I_{N}(\gamma)=\frac{I(\gamma)}{I(0)}, \quad \gamma=\frac{b_{3}-b_{2}}{b_{4}-b_{2}},
$$

where $I(\gamma)$ is the steady-state biosensor current calculated at given level $\gamma$ of the enzyme filling. The filling level $\gamma$ can also be called as the relative volume of the enzyme in the holes. Figure 3 shows the results of calculation.
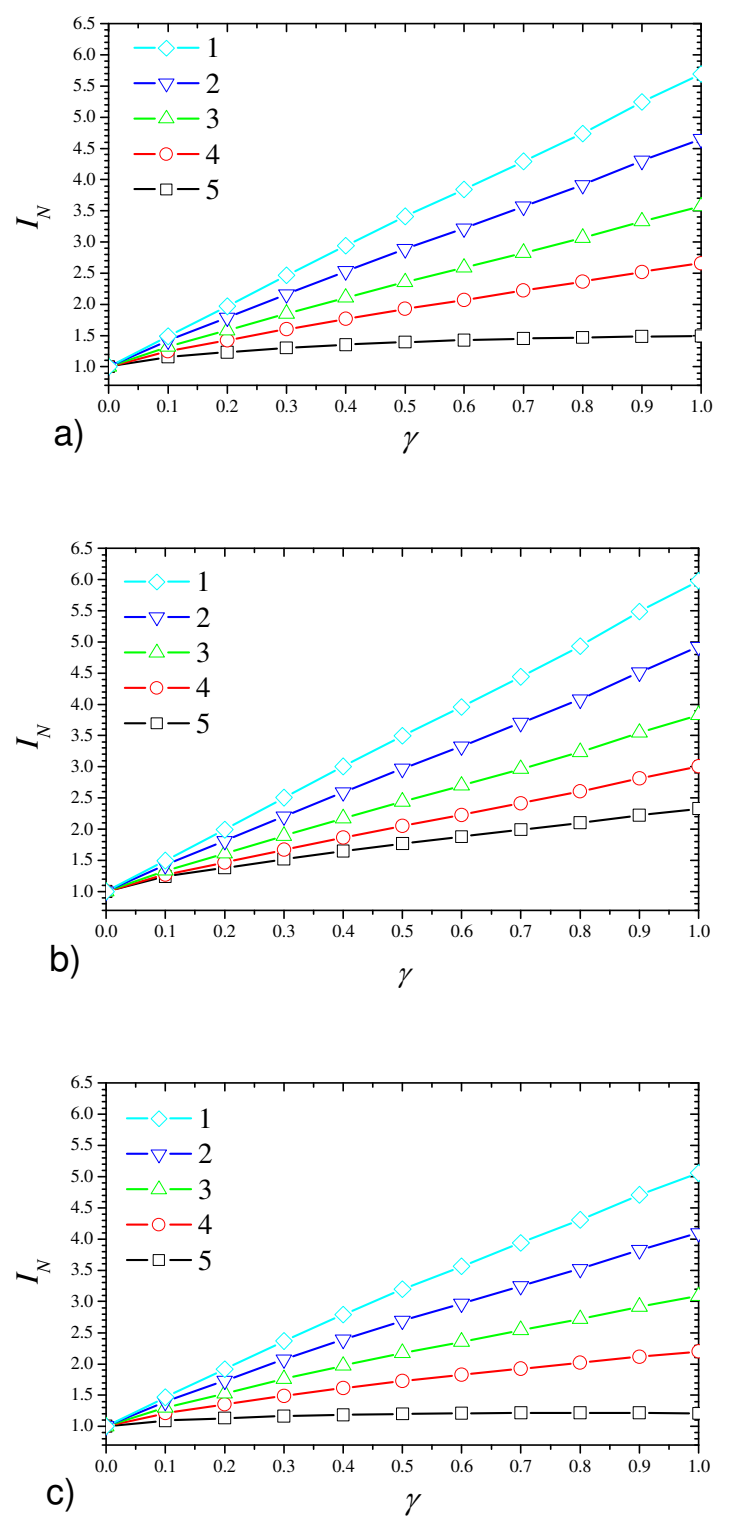

Figure 3: The normalized steady-state current $I_{N}$ versus the filling level $\gamma$ of the enzyme in holes at different values of the radius $a_{1}$ of holes, $a_{1}=1.0$ (1), 0.8 (2), 0.6 (3), 0.4 (4), 0.2 (5) $\mu \mathrm{m}, S_{0}=100$ (a, b), 1 (c) $\mu \mathrm{M}, V_{\max }=$ $100(\mathrm{a}, \mathrm{c}), 1$ (b) $\mu \mathrm{M} / \mathrm{s}, a_{2}=1, b_{1}=2, b_{2}=4, b_{4}=14, b_{5}$ $=16 \mu \mathrm{m}, K_{M}=100 \mu \mathrm{M}, D_{1}=1, D_{2}=300, D_{3}=600$ $\mu \mathrm{m}^{2} / \mathrm{s}$. 
One can see in Figure 3, the steady state biosensor is a monotonous increasing function of the level $\gamma$ of the enzyme filling. The behaviour of the biosensor response significantly depends on the radius $a_{1}$ of the holes, the maximal enzymatic rate $V_{\max }$ and substrate concentration $S_{0}$. In the cases of relatively small radius $a_{1}$ of the holes of the perforated membrane $\left(a_{1} \leq 0.2 a_{2}\right)$ and high the maximal enzymatic rate $\left(V_{\max }=100 \mu \mathrm{M} / \mathrm{s}\right)$ the steady state biosensor current practically does not depend on the level $\gamma$ of holes filling with enzyme (Figures 3a and $3 c$ ). Since in practice, it is difficult to ensure fully enzyme-filled holes, this feature of the biosensors can be applied in design of novel biosensors having response stable to changes in the level of holes filling.

In the case of relatively large radius $a_{1}$ of holes $\left(a_{1} \geq 0.4\right.$ $a_{2}$ ) the biosensor response is very sensitive to changes of the holes filling. The response sensitivity to changes of $\gamma$ also increases with decrease of the enzymatic activity $V_{\max }$ (Figure 3b). The effect of the substrate concentration is rather low for a wide rages of maximal enzymatic rate $V_{\max }$.

\section{CONCLUSIONS}

The mathematical model of an operation of the amperometric biosensors with selective and perforated membranes can be used to investigate peculiarities of the biosensor response. The model can be applied for modelling the response of the biosensors when the holes in the perforated membrane are partially filled with an enzyme.

The steady state biosensor current is a monotonous increasing function of the filling level $\gamma$ of the enzyme in holes (Figure 3).

In the case of relatively small radius of holes and high enzymatic activity the biosensor response practically does not depend upon the level of filling the holes with the enzyme. Otherwise, the filling level should be taken into consideration when modelling the biosensor action accurately (Figure 3).

\section{ACKNOWLEDGEMENTS}

The author expresses sincere gratitude to prof. Feliksas Ivanauskas and prof. Juozas Kulys for their valuable contribution to modelling of biosensors.

\section{REFERENCES}

Baeumner, A. J.; C. Jones; C.Y. Wong and A. Price. 2004. “A Generic Sandwich-type Biosensor with Nanomolar Detection Limits". Analytical and Bioanalytical Chemistry 378, No. 6, 1587-1593.

Baronas, R.; F. Ivanauskas and J. Kulys. 2003. "The Influence of the Enzyme Membrane Thickness on the Response of Amperometric Biosensors". Sensors 3, No. 7, 248-262.
Baronas, R.; F. Ivanauskas and J. Kulys. 2004. "The Effect of Diffusion Limitations on the Response of Amperometric Biosensors with Substrate Cyclic Conversion". Journal of Mathematical Chemistry, 35, No. 3, 199-213.

Baronas, R.; J. Kulys and F. Ivanauskas. 2006. "Computational Modelling of Biosensors with Perforated and Selective Membranes". Journal of Mathematical Chemistry 39, No. 2, 345-362.

Britz, D. 1988. Digital Simulation in Electrochemistry, 2nd ed. Springer-Verlag, Berlin.

Ferreira, L. S.; M.B. De Souza; J.O. Trierweiler; O. Broxtermann; R.O.M. Folly and B. Hitzmann. 2003. "Aspects Concerning the Use of Biosensors for Process Control: Experimental and Simulation Investigations. Computers and Chemical Engineering 27, No. 8-9, 11651173.

Moreira, J.E.; S.P. Midkiff; M. Gupta; P.V. Artigas; M. Snir and R.D. Lawrence. 2000. "Java programming for high performance numerical computing". IBM Systems Journal $39,21-56$.

Rogers, K.R. 1995. "Biosensors for Environmental Applications". Biosensors and Bioelectronics 10, No. 6-7, 533-541.

Samarskii, A.A. 2001. The Theory of Difference Schemes. Marcel Dekker, New York-Basel.

Scheller, F. and F. Schubert. 1992. Biosensors. Elsevier Science, Amsterdam.

Schulmeister, T. and D. Pfeiffer. 1993. "Mathematical Modelling of Amperometric Enzyme Electrodes with Perforated Membranes. Biosensors Bioelectronics 8, No. 2, 75-79.

Turner, A.P.F.; I. Karube and G.S. Wilson. 1987. Biosensors: Fundamentals and Applications. Oxford University Press, Oxford.

Wollenberger, U., F. Lisdat and F.W. Scheller. 1997. Frontiers in Biosensorics 2: Practical Applications. Birkhauser Verlag, Basel.

\section{AUTHOR BIOGRAPHY}

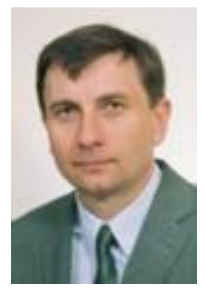

ROMAS BARONAS was born in 1959 in Kybartai, Lithuania. He enrolled at the Vilnius University, where he studied applied mathematics and received his $\mathrm{PhD}$ degree. Now he is a Professor in the Department of Software Engineering at the Vilnius University. He also joined the Software Engineering Department at the Institute of Mathematics and Informatics. His research interests are scientific computation, database systems and software engineering. His e-mail address is: romas.baronas@mif.vu.lt and his Web-page can be found at http://www.mif.vu.lt/ baronas. 